\title{
Screening the Coulomb interaction and thermalization of Anderson insulators
}

\author{
Z. Ovadyahu
}

\author{
Racah Institute of Physics, The Hebrew University, Jerusalem 91904, Israel
}

\begin{abstract}
Long range interactions are relevant for a wide range of phenomena in physics where they often present a challenge to theory. In condensed matter, the interplay of Coulomb interaction and disorder remains largely an unsolved problem. In two dimensional films the long-range part of the Coulomb interaction may be screened by a nearby metallic overlay. This technique is employed in this work to present experimental evidence for its effectiveness in limiting the spatial range of the Coulomb interaction. We use this approach to study the effects of the long-range Coulomb interaction on the out-of-equilibrium dynamics of electron-glasses using amorphous indium-oxide films. The results demonstrate that electronic relaxation times, extending over thousands of seconds, do not hinge on the long-range Coulomb interaction nor on the presence of a real gap in the density of states. Rather, they emphasize the dominant role played by disorder in controlling the slow thermalization processes of Anderson insulators taken far from equilibrium.

PACS numbers: 72.15.Rn 61.43.-j
\end{abstract}

\section{INTRODUCTION}

The interplay between disorder and Coulomb interaction has been a challenging problem in condensed matter physics. Effects associated with disorder while neglecting interaction may still be a difficult problem to solve. Such theories however are rarely applicable for experiments as disorder and interactions appear to be connected; increasing one usually increases the other. Few comprehensive studies treating disorder and interactions were made, usually when both are fairly weak or when the spatial range of the interaction is limited. In the strongdisorder regime however, neglecting the long-range part of the interaction is difficult to justify, thus further compounding a difficult problem. This is true in particular for the Anderson localization case where the question of Coulomb interaction originated decades ago [1, 2] is still unsolved despite extensive efforts. Some progress has been made on this many-body problem for short-range interaction [3-5] but effects of the long-range component are yet largely unresolved.

An intriguing result of the disorder-interaction competition is the appearance of a non-ergodic phase exhibiting glassy features. These involve slow conductancerelaxations of Anderson insulators taken far from the equilibrium and a variety of memory effects [6, 7]. Relaxation times that extend over thousands of seconds are observable at temperatures where the hopping-length, which is the effective screening-length in the insulating regime, is of the order of $20 \mathrm{~nm}$.

Theoretical models that qualitatively account for these effects are based on the opening up of a soft-gap 8 - 15 in the system density of states (DOS). This, so called Coulomb-gap [16], is reflected in the conductance $G$ versus gate-voltage $V_{g}$ as a cusp-like minimum centered at the point where the system was allowed to relax (the 'memory-dip') [7]. To be observable in $G\left(V_{g}\right)$ scans, $\partial \mathrm{V}_{\mathrm{g}} / \partial \mathrm{t}$ must be fast enough relative to the relaxation- rate of the electronic system [17]. For technical reasons this condition limits the choice of systems to Andersoninsulators with relatively high carrier-concentration $N$ where both disorder and interactions are strong [17]. The importance of strong disorder and interaction is attested by the seven different systems that exhibit these nonequilibrium effects all sharing the feature of high carrierconcentration $N \gtrsim 10^{19} \mathrm{~cm}^{-3}$. It is yet not clear however what role is played by the long-range Coulomb interaction in these phenomena.

In this work we attempt to find answers to this and related questions by using a metallic ground-plane in proximity to the sample to modify the long-range Coulomb interaction in a controlled way. Using samples configured for field-effect measurements, and furnished with a nearby screening-plane, yield results consistent with the anticipated [18] outcome for a modified Coulomb-gap. The dynamics of these systems, on the other hand, does not show significant difference relative to the reference samples. It seems therefore that, in addition to strong enough quenched disorder, short and medium-range interactions may be sufficient to account for the long relaxation times observed in the experiments. In particular, the results demonstrate that relaxation times extending over hours are sustainable in interacting Anderson insulators even while having a finite density-of-states at the chemical potential.

To optimize the effect of screening by a nearby metal, the system chosen for the study had rather low carrierconcentration. This also resulted in systems with short relaxation-times. We took advantage of the latter to systematically study the deviation from the logarithmic relaxation-law to elucidate the relative importance of disorder and interaction to the slow dynamics of the glassy phase. 


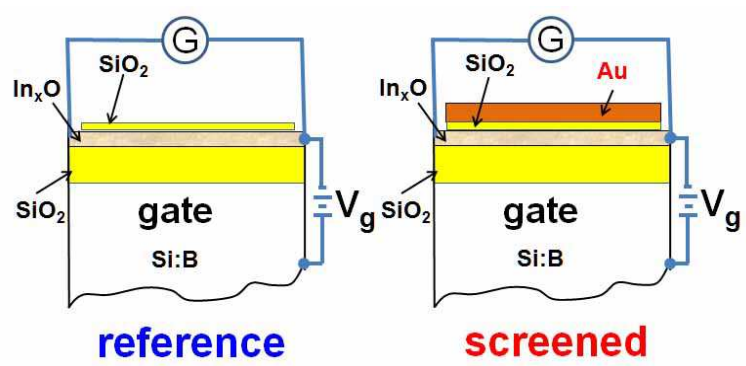

FIG. 1: Schematic description of the reference and screened samples configured for field-effect measurements.

\section{EXPERIMENTAL}

\section{Sample preparation and measurement techniques}

Samples used in this study were $200 \AA$ thick films of $\operatorname{In}_{\mathrm{x}} \mathrm{O}$. These were made by e-gun evaporation of $99.999 \%$ pure $\mathrm{In}_{2} \mathrm{O}_{3}$ onto room-temperature $\mathrm{Si}$ wafers in a partial pressure of $1.5 \times 10^{-4} \mathrm{mBar}$ of $\mathrm{O}_{2}$ and a rate of $0.5 \pm 0.1 \AA / \mathrm{s}$. The Si wafers (boron-doped with bulk resistivity $\rho \leq 2 \times 10^{-3} \Omega \mathrm{cm}$ ) were employed as the gateelectrode in the field-effect and gate-excitation experiments. The samples were deposited on a $\mathrm{SiO}_{2}$ layer $(2 \mu \mathrm{m}$ thick) that was thermally-grown on these wafers and acted as the spacer between the sample and the conducting Si:B substrate.

The as-deposited films had sheet-resistance $R_{\square}>G \Omega$ at room-temperature. They were then thermally-treated. This was done by stages; the samples were held at a constant temperature starting from $\approx 340 \mathrm{~K}$ for $20-30$ hours then the temperature was raised by $5-10 \mathrm{~K}$ for the next stage. This was repeated until the desired $\mathrm{R}_{\square}$ was attained (see [19] for fuller details of the thermalannealing and structure analysis). This process yielded samples with $\mathrm{R}_{\square}=18-45 \mathrm{k} \Omega$ that at $\mathrm{T} \approx 4 \mathrm{~K}$ spanned the range of $100 \mathrm{k} \Omega$ to $40 \mathrm{M} \Omega$. The carrier-concentration $N$ of these samples, measured by the Hall-Effect at room-temperatures, was in the range $N=8.7 \times 10^{18} \mathrm{~cm}^{-3}$ to $2 \times 10^{19} \mathrm{~cm}^{-3}$.

The main focus in this work was a study of the effects produced by screening the long range part of the Coulomb interaction on the nonequilibrium transport of Anderson insulators. The experimental methodology we employed is a comparing simultaneously deposited samples, placing a metallic-plane in close proximity to just one of them. Figure 1 illustrates the geometry of the pair of samples, labeled "screened" and "reference", each configured for field-effect measurements.

The distance between the screened sample and the screening-layer ( $\mathrm{a} \approx 200 \AA$ gold film) is determined by the thickness $d$ of the $\mathrm{SiO}_{2}$ layer. This spacer, 7$11 \mathrm{~nm}$ thick, was e-gun deposited on both the screened and reference sample simultaneously using pure quartz as the source. The Anderson insulator that was chosen for these experiments was the version of $\operatorname{In}_{\mathrm{x}} \mathrm{O}$ with low carrier-concentration $\left(N \leq 2 \times 10^{19} \mathrm{~cm}^{-3}\right)$. This version has several attractive features for these experiments: In the first place, the relatively large inter-carrier distance $N^{-1 / 3} \simeq 5 \mathrm{~nm}$, allows the spacer d to be thick enough to minimize pinholes while $\mathrm{d} N^{1 / 3}$ may still be small enough for effective screening. Secondly, the electron-glass dynamics becomes faster as their carrier-concentration falls below $N \lesssim 4 \times 10^{19} \mathrm{~cm}^{-3}$ while, all other things being equal, the relative value of the excess-conductance in the excited state $\Delta \mathrm{G} / \mathrm{G}$ is more conspicuous than in samples with $N>4 \mathrm{x} 10^{19} \mathrm{~cm}^{-3}$. These expectations were borne out in our experiments which made it possible to quantify the system dynamics as it approaches the quantum phase transition.

Conductivity of the samples was measured using a twoterminal ac technique employing a 1211-ITHACO current preamplifier and a PAR-124A lock-in amplifier. Measurements were performed with the samples immersed in liquid helium at $\mathrm{T} \approx 4.1 \mathrm{~K}$ held by a 100 liters storagedewar. This allowed up to two months measurements on a given sample while keeping it cold. These conditions are essential for the measurements described below where extended times of relaxation processes are required at a constant temperature, especially when running multiple excitation-relaxation experiment on the same sample.

The gate-sample voltage (referred to as $\mathrm{V}_{\mathrm{g}}$ in this work) in the field-effect measurements was controlled by the potential difference across a $10 \mu \mathrm{F}$ capacitor charged with a constant current fed by the Keithley K220. The rate of change of $\mathrm{V}_{\mathrm{g}}$ is determined by the value of this current. The range of $\mathrm{V}_{\mathrm{g}}$ used in this study reached in some cases $\pm 50 \mathrm{~V}$ which is equivalent to the $\pm 12 \mathrm{~V}$ used in previous studies where the gate-sample separation was $0.5 \mu \mathrm{m}$ as compared with the $2 \mu \mathrm{m} \mathrm{SiO}_{2}$ spacer used here.

The ac voltage bias in conductivity measurements was small enough to ensure near-ohmic conditions. The voltage used in the relaxation experiments was checked to be in the linear response regime by plotting the currentvoltage characteristics of each sample.

\section{RESULTS AND DISCUSSION}

\section{Modifying the memory-dip by a screening-plane}

The idea behind the use of the elaborate construction described in Fig.1 was to find out the effect of eliminating (or at least weakening) the long-range part of the Coulomb interaction. This relies on comparing results of identical measurements on the screened and reference samples. For that to be a tenable procedure, one has to ascertain that the two samples differ only by the imagecharges created in the nearby gold layer. This is not a trivial undertaking to secure as the act of depositing 


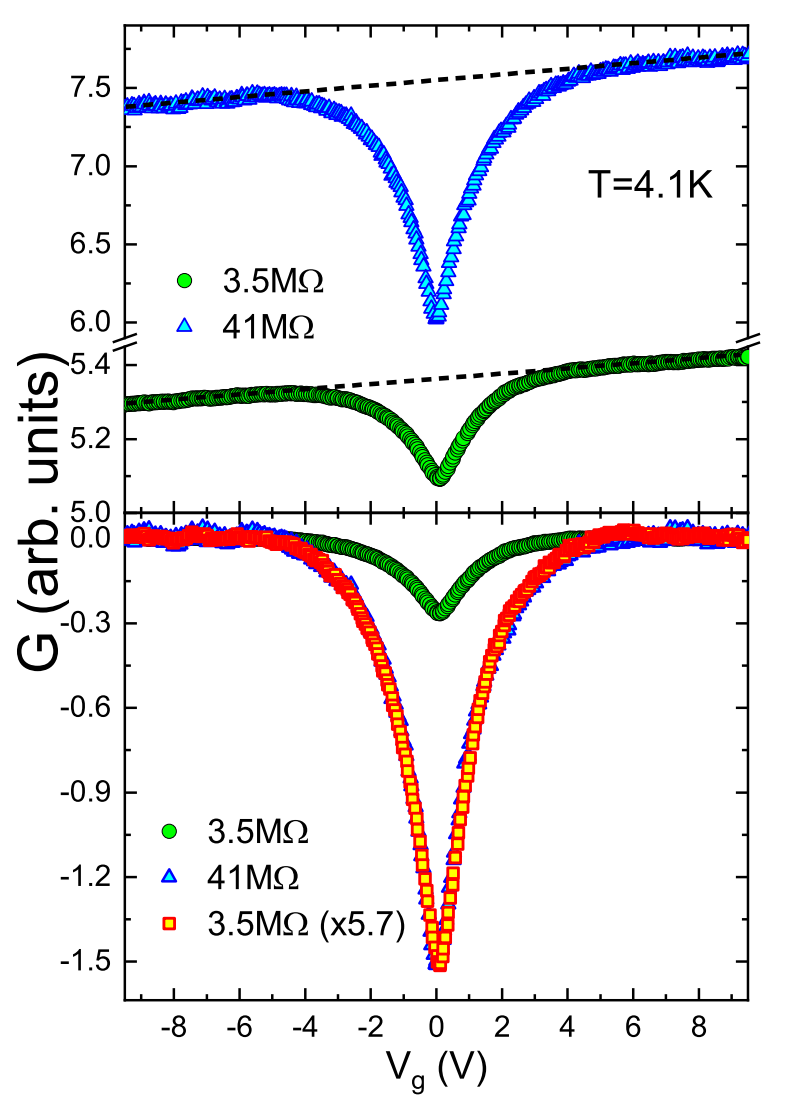

FIG. 2: Field-effect plots $\mathrm{G}\left(\mathrm{V}_{\mathrm{g}}\right)$ for two $\operatorname{In}_{\mathrm{x}} \mathrm{O}$ samples prepared from the same evaporation batch $\left(N=8.7 \times 10^{19} \mathrm{~cm}^{-3}\right)$ but subjected to different thermal-annealing (top plate). The dashed lines depict the thermodynamic component of the respective $G\left(V_{g}\right)$. The bottom plate shows the MD's of these samples (after subtracting their respective thermodynamic component), and demonstrate that their magnitude may be made to scale just by a multiplicative constant.

the gold layer may inadvertently break the symmetry between the reference and screened samples; For example, the heat produced during deposition of the gold layer will unavoidably cause some annealing in the screened sample. A different disorder in the screened sample may also arise from the strain related to mismatch in mechanical properties of the $\mathrm{Au} / \mathrm{SiO}_{2}$ interface. In principle, a difference in disorder between the screened-reference samples can be compensated by a judicious thermal annealing of the samples to make their room-temperature resistivity close to one another. However, being Anderson insulators, a few percent difference in room-temperature resistance may translate to orders of magnitude disparity at liquid helium temperatures.

Fortunately, the feature that is targeted for investigation here is not susceptible to these artifacts; The shape of the memory-dip which reflects the underlying Coulomb-gap is a robust feature. At a given temperature, the MD shape is independent of the sample disorder, the sweep-rate, time since cooldown, magnetic-fields etc., it

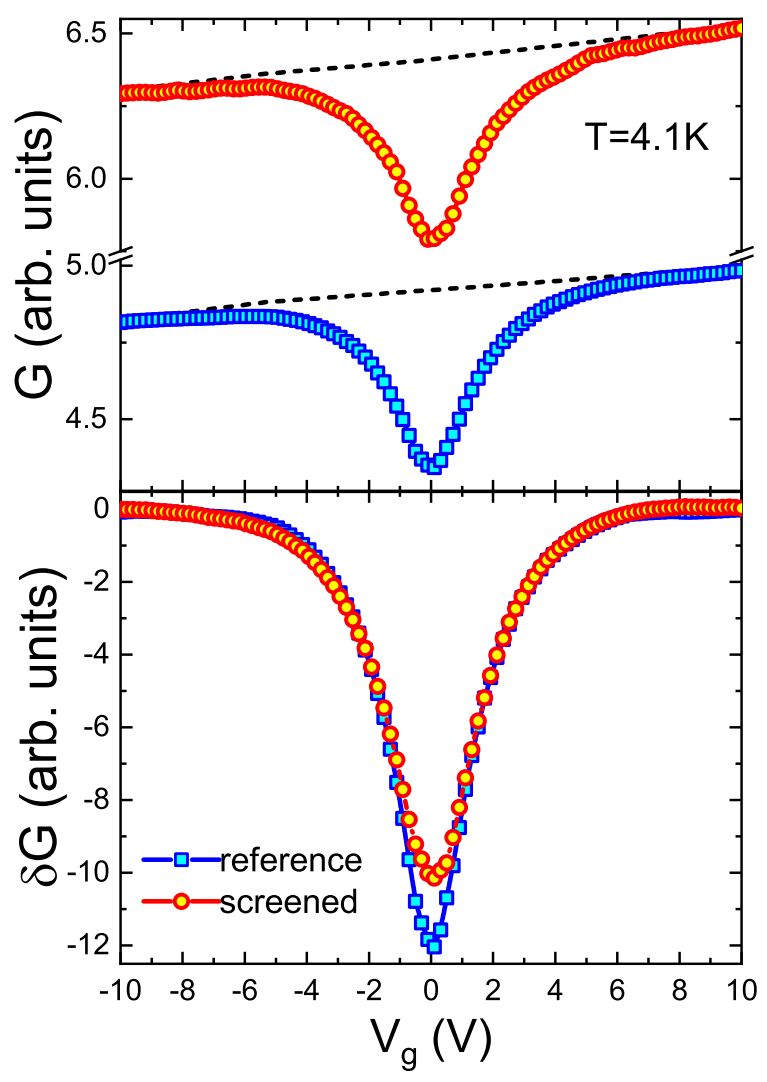

FIG. 3: Field-effect plots comparing $G\left(V_{g}\right)$ for a reference (squares) vs. screened (circles) $\operatorname{In}_{\mathrm{x}} \mathrm{O}$ samples from the same evaporation batch as in Fig.2. The bottom plate is an attempt to scale the MD's by a constant factor showing a reasonable fit for the "wings" but the screened-MD falls short of the reference-MD for the bottom part of the $G\left(V_{g}\right)$.

depends only on the carrier-concentration which is set by the $\mathrm{In} / \mathrm{O}$ ratio as demonstrated in [7]. To illustrate, figure 2 shows the dependence of the conductance $\mathrm{G}$ on gate-voltage $\mathrm{V}_{\mathrm{g}}$ for two of the studied 'reference' samples. These share the same composition but were subjected to different degree of annealing and thus exhibit different sheet-resistance (and thus disorder).

The top plate of Fig.2 show the raw data for the fieldeffect $G\left(V_{g}\right)$ of these samples. Two features are observed in this figure; an asymmetric component characterized by $\partial \mathrm{G}\left(\mathrm{V}_{\mathrm{g}}\right) / \partial \mathrm{V}_{\mathrm{g}}>0$ that reflects the increased thermodynamic density-of-states with energy (the thermodynamic field-effect), and a cusp-like dip centered at $\mathrm{V}_{\mathrm{g}}=0$ where the system was allowed to relax before sweeping the gate voltage (the memory-dip). By subtracting from each plot the respective thermodynamic $\mathrm{G}\left(\mathrm{V}_{\mathrm{g}}\right)$ component, one gets the two MD's that, after multiplication by a constant are shown to have the same shape despite the large disparity in their resistance.

By contrast, the MD's of the reference-screened samples fail to show similar data collapse. Figure 3 shows the results of an attempt to match the memory-dips 

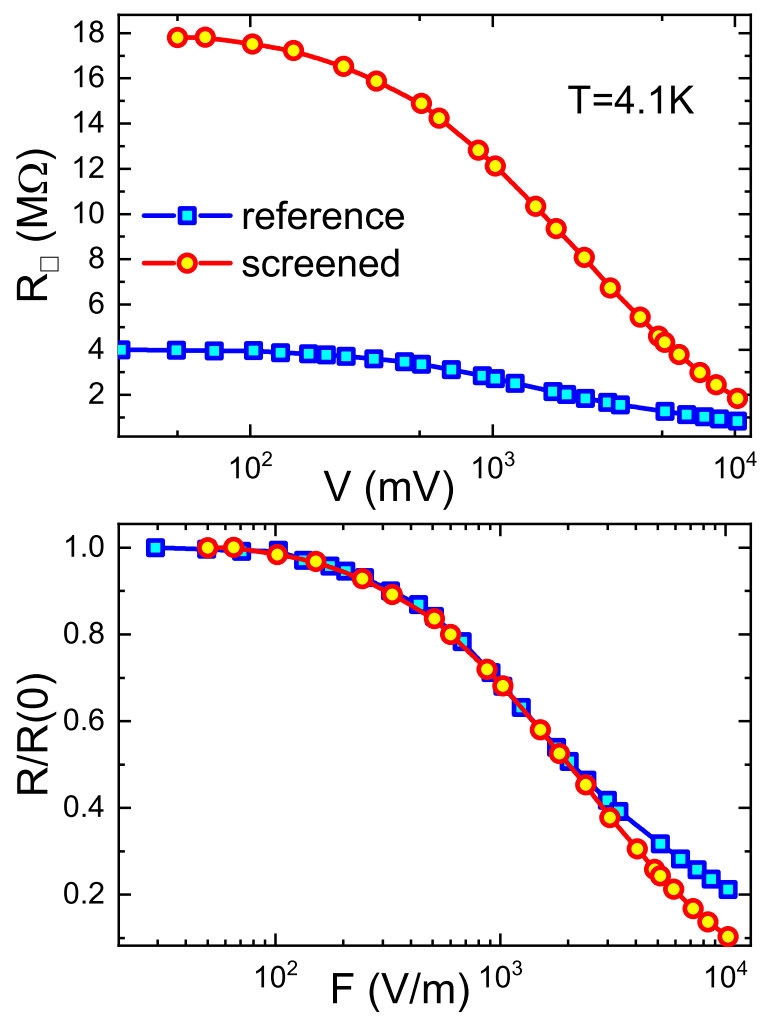

FIG. 4: Top plate: The dependence of the sheet-resistance $\mathrm{R}_{\square}$ of the screened (circles) and reference (squares) samples on the applied voltage (same samples as in Fig.3). Bottom plate: The relative change of these resistances showing a similar functional dependence.

for a specific couple. In this case, factor-scaling the data for the two memory-dips is possible for most of the range of $G\left(V_{g}\right)$ but not near its equilibrium point where the screened dip falls short of the reference. Figure 4 shows however that the current-field characteristics of these samples is nearly identical even deeper into the non-ohmic regime and there is no sign of a current-short from the active sample to the screening-layer.

Note that in this pair, the resistance of the screened sample was larger than that of the reference. The cutback-shaped MD of the screened sample relative to the reference has been observed in all six pairs studied in this work, This was independent of the relative value of the resistances involved. The scaled results for a pair where the sheet-resistance $\mathrm{R}_{\square}$ of the screened sample is smaller than that of the reference sample are shown in Fig.5 which depicts the same qualitative features as in Fig.3. Finally, Fig.6 shows two more referencescreened pairs taken from a single specific depositionbatch with carrier-concentration $N \approx 1.9 \times 10^{19} \mathrm{~cm}^{-3}$. The figure includes both $\mathrm{R}_{\square}$ (reference) $>\mathrm{R}_{\square}$ (screened) and $\mathrm{R}_{\square}$ (reference) $<\mathrm{R}_{\square}$ (screened) cases as well as an extended range of the field-effect vs. a higher resolution view of the memory-dip main features. Screening by

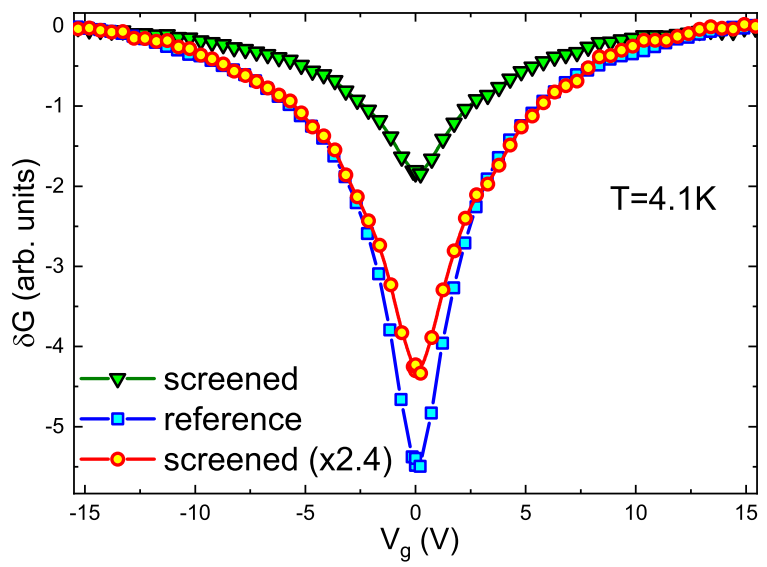

FIG. 5: Comparing the MD shape of a reference sample with $\mathrm{R}_{\square}=6.3 \mathrm{M} \Omega$ with a screened sample of the same deposition batch with $\mathrm{R}_{\square}=3.1 \mathrm{M} \Omega$.

a nearby metallic plane has been shown to change the spatial dependence of the Coulomb interaction even in diffusive systems; Some features of the single-particle DOS found in tunneling experiments on two and threedimensional indium-oxide samples differed in both magnitude and functional dependence from those predicted by simple models of interaction and disorder [20]. These differences were accounted for by Altshuler et al based on the image-charges created due to proximity of the sample to the tunneling electrode [21]. The lack of screening in the Anderson-insulating phase makes the system more susceptible to the influence of the nearby metallic-plane. A modified form of the Coulomb interaction is therefore an expected effect 22]. Indeed, the reduced relative magnitude of the screened-MD observed in the current experiments is consistent with the effect of a screening layer on the Coulomb-gap of a 2D system. This effect was estimated theoretically by Hadley et al [18]. In our six samples the reduced magnitude of the MD of the screened sample ranged between $\approx 12 \%$ to $\approx 23 \%$, which according to [18] are associated with $\mathrm{d} N^{1 / 3} \approx 2.8$ to $\approx 1.6$ respectively. For the carrier-concentrations used in this work $N=8.7 \times 10^{19} \mathrm{~cm}^{-3}$ to $1.9 \times 10^{19} \mathrm{~cm}^{-3}$, these values give $\mathrm{d}$ in the range $8-15 \mathrm{~nm}$ which in good agreement with the thickness of the $\mathrm{SiO}_{2}$ spacer used (see section II above). In the six pairs of screened-reference samples however, it was not possible to see a systematic dependence on the spacer $d$. This is probably due to relatively large thickness variations in these thin films; both $\mathrm{SiO} 2$ and $\operatorname{In}_{\mathrm{x}} \mathrm{O}$ have been tested by AFM which showed thickness fluctuations of the order of $\pm 8 \%$ [23].

It is natural to ask how limiting the interaction-range affects how the system thermalizes after being taken out of equilibrium, attempts to answer this question are discussed next. 

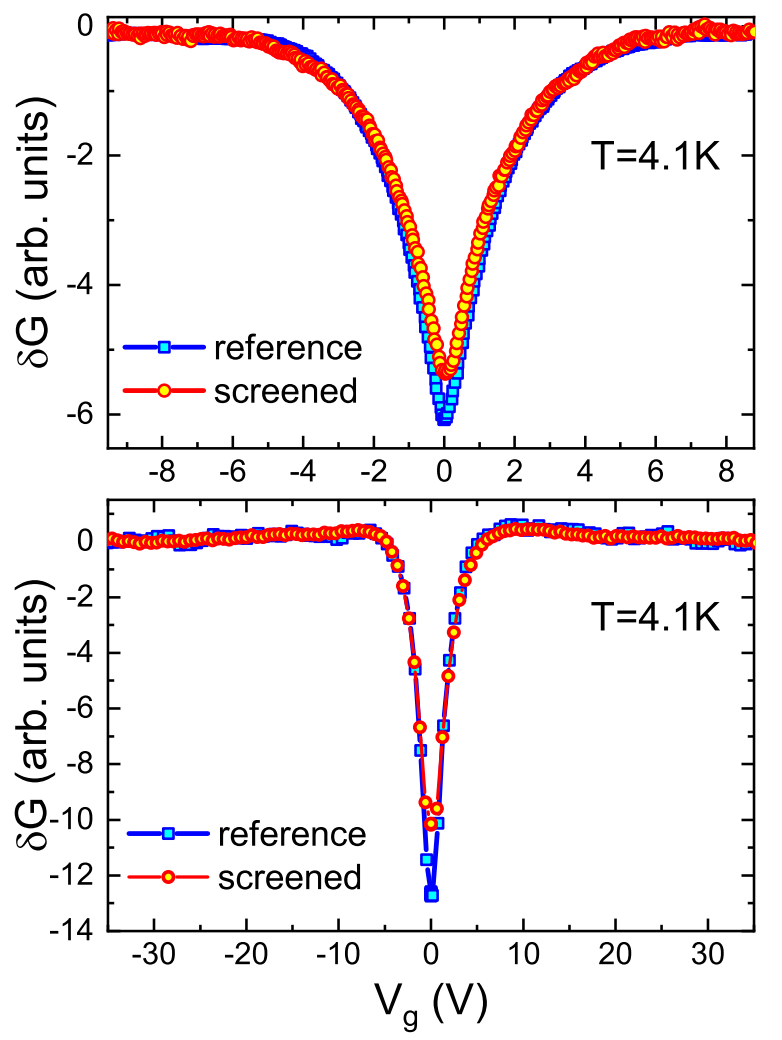

FIG. 6: Attempting to scale the functional dependence of the MD of a reference-screened pair of samples from the same deposition batch (with $N \approx 1.9 \times 10^{19} \mathrm{~cm}^{-3}$ ). Top plate: reference sample with $R_{\square}=4 \mathrm{M} \Omega$, and screened sample with $R_{\square}$ $=40 \mathrm{M} \Omega$. Bottom plate: reference sample with $\mathrm{R}_{\square}=6.7 \mathrm{M} \Omega$; screened sample with $\mathrm{R}_{\square}=45 \mathrm{M} \Omega$.

\section{Thermalization dynamics}

\section{Experimental definition of the thermalization-time}

It is rarely possible to ascertain experimentally that a system under observation is thermalized. One may however monitor the process of the approach towards equilibrium by following a specific measurable and associate the state of thermalization with the time where this measured quantity reached a time-independent value relative to which the system just fluctuates. Thermalization and relaxation will be used here interchangeably although technically the time-independent regime may only signal pre-thermalization.

An effective and way to take the system far from equilibrium and observe the ensuing relaxation is the 'gateprotocol'. In this protocol a nonequilibrium state is created by switching the gate-voltage $\mathrm{V}_{\mathrm{g}}$ from an equilibrium value $V_{\text {eq }}$ to a new one $V_{n}$. This process is reflected in the appearance of excess-conductance $\Delta \mathrm{G}(\mathrm{t})$ that decays slowly with time. An example for the results obtained with this protocol is shown in Fig.7.

The relaxation to the equilibrium under the newly

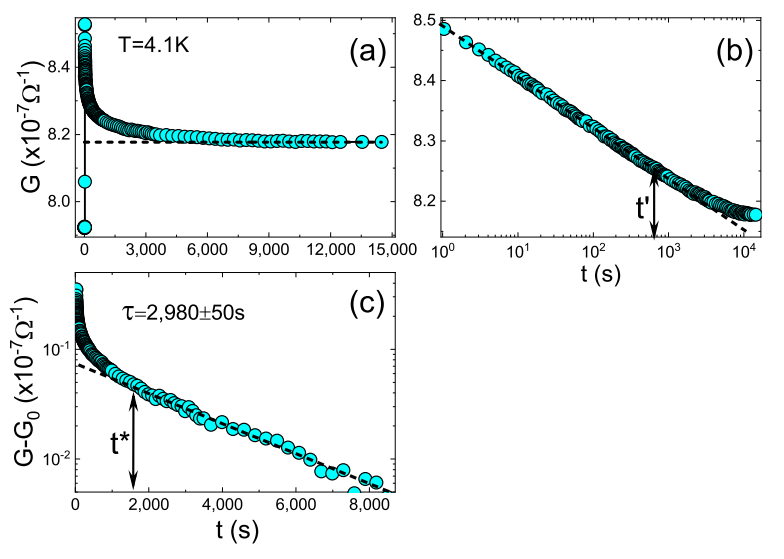

FIG. 7: Results of using the gate-protocol (see text) on a sample with $\mathrm{R}_{\square}=1.2 \mathrm{M} \Omega$. (a) Conductance as function of time; after $\approx 30$ seconds of monitoring $\mathrm{G}$ under $\mathrm{V}_{\mathrm{g}}=0 \mathrm{~V}$ the gate-voltage was swept to $\mathrm{V}_{\mathrm{g}}=40 \mathrm{~V}$ at a rate of $15 \mathrm{~V} / \mathrm{s}$. The dashed line is $\mathrm{G}_{0}$, the asymptotic value of the conductance. $\mathrm{G}_{0}=\mathrm{G}(40 \mathrm{~V})$ differs from $\mathrm{G}\left(\mathrm{V}_{\text {eq }}\right)$ due to the component of the thermodynamic field-effect. (b) Conductance relaxation starting from the time $\mathrm{V}_{\mathrm{g}}=40 \mathrm{~V}$ was established showing the extent of the $\log (\mathrm{t})$ dependence (delineated by the dashed line). $t$ ' marks the point where $G(t)$ deviates from the logarithmic dependence. (c) The plot of $\mathrm{G}(\mathrm{t})-\mathrm{G}_{0}$ demonstrating an exponential relaxation law: $\Delta \mathrm{G}(\mathrm{t}) \propto \exp [-(\mathrm{t} / \tau)]$ (dashed line is best fit yielding the relaxation time $\tau$ for the sample). $t^{*}$ marks the time below which $G(t)$ deviates from exponential-relaxation.

established $\mathrm{V}_{\mathrm{n}}$ was monitored through the measured $\Delta \mathrm{G}(\mathrm{t})$. As observed in Fig.7, $\Delta \mathrm{G}(\mathrm{t}) \propto-\log (\mathrm{t})$ for several hundred seconds (up to t' in Fig.7b), and after a time marked as $\mathrm{t}^{*}$ (Fig.7c) the relaxation-law reverts to $\Delta \mathrm{G}(\mathrm{t}) \propto \exp [-\mathrm{t} / \tau]$, which defines $\tau$ that will be used here as the characteristic thermalization-time. As will be shown in the next paragraph, when properly implemented, this 'gate-protocol' is equivalent to quenchcooling the system from high temperatures. The latter, has the advantages of being history-free but the thermalcycle runs the risk of changing the structure of the sample (and possibly damage it more seriously), and it also sacrifices the short-time relaxation because one must wait for the sample and its surroundings (sample stage, thermometer, etc.) to cool to the bath temperature. The gate-protocol, by contrast, may be safely repeated many times on the same sample and no 'parasitic' heating is involved in the process.

An important caveat when using the gate-protocol is to let the sample reach equilibrium before changing the gate voltage to a new value to avoid history dependence [24]. As a check on this point, we compared the relaxation time of a sample by both, a thermal-quench and the gate-protocol. The results, shown in Fig.8, demonstrate that the relaxation-time $\tau$ based on the gate-protocol is essentially identical with that based on quench-cooling the sample. We believe that the gate-protocol can be 

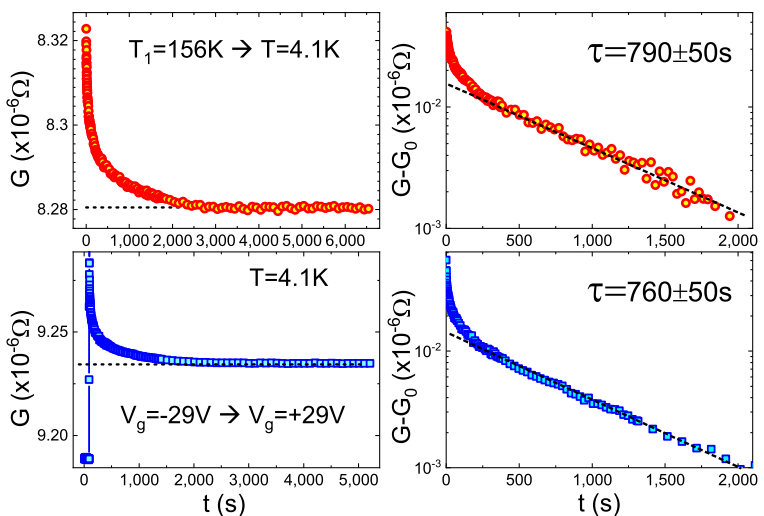

FIG. 8: The relaxation dynamics of a $\operatorname{In}_{x} \mathrm{O}$ film with $\mathrm{R}_{\square}$ $=0.13 \mathrm{M} \Omega$ at $4.1 \mathrm{~K}$ and $N \approx 1.9 \times 10^{19} \mathrm{~cm}^{-3}$ tested by two protocols: Top two plates: Using a quench-cool protocol. Lower two plates: Using the gate protocol. Dashed lines delineate the equilibrium conductance $\mathrm{G}_{0}$ for each protocol. The relaxation time $\tau$ is obtained from fit to $\Delta \mathrm{G}(\mathrm{t}) \propto \exp [-(\mathrm{t} / \tau)]$ for the data in the two right-hand-side plots.

relied upon to yield the correct relaxation-time provided the equilibration-time is longer than $\tau$. For the series of measurements reported below, the samples were equilibrated under $\mathrm{V}_{\text {eq }}$ for at least 12 hours under $\mathrm{V}_{\text {eq }}$.

\section{Dynamics of screened-reference samples}

Comparing between screened and the reference samples is more problematic when it comes to dynamics than the difficulties mentioned above with regard to the effect on the shape of the memory-dip. The latter is independent of the disorder; the MD shape is the same even when the sample $\mathrm{R}_{\square}$ changes by order of magnitude (see Fig.2 above) while the dynamics is quite sensitive to the sample disorder [17] as will be demonstrated below. Figure 9 shows $\Delta \mathrm{G}(\mathrm{t})$ for the asymptotic relaxation regime generated by using the gate-protocol. These data were taken on the same samples used for comparing the MD shapes in Fig.5 above that, in terms of their $\mathrm{R}_{\square}$, is our best-matched screened-reference pair. The data in Fig.9 clearly suggest that the relaxation-time of the screened sample is essentially the same as the reference. Therefore, limiting the range of the Coulomb interaction to $\approx 8 \mathrm{~nm}$ does not have a significant effect on the system relaxation-time. Moreover, $\tau$ of the order of few thousands of seconds is manifestly possible even without longrange interaction. This is a useful piece of information that should make it easier for theory to finally address the long-standing question of the slow relaxation times of some electron-glasses 24. We return to this issue after discussing the results of the dynamics as function of disorder.

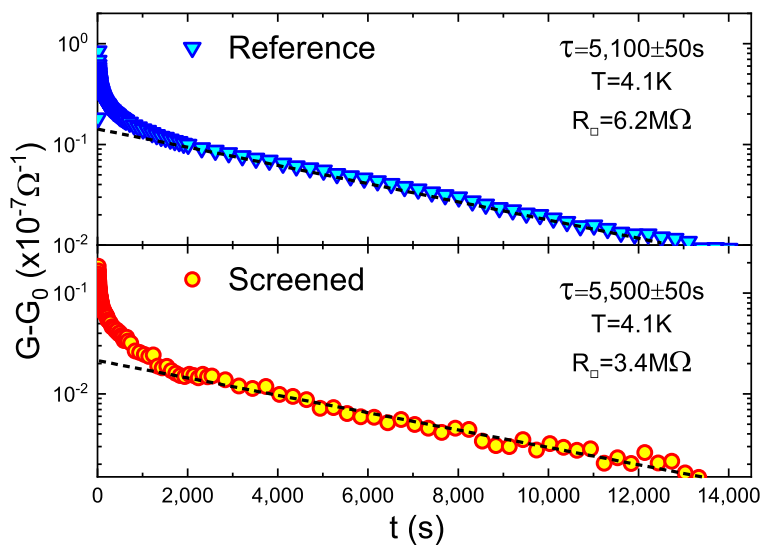

FIG. 9: The asymptotic relaxation curves for a specific $\operatorname{In}_{\mathrm{x}} \mathrm{O}$ screened-reference pair $\left(N \approx 1.9 \times 10^{19} \mathrm{~cm}^{-3}\right)$ using the gate protocol under identical conditions. Dashed lines are best fits to $\Delta \mathrm{G}(\mathrm{t}) \propto \exp [-(\mathrm{t} / \tau)]$ that yielded the respective relaxation time shown in the figures.

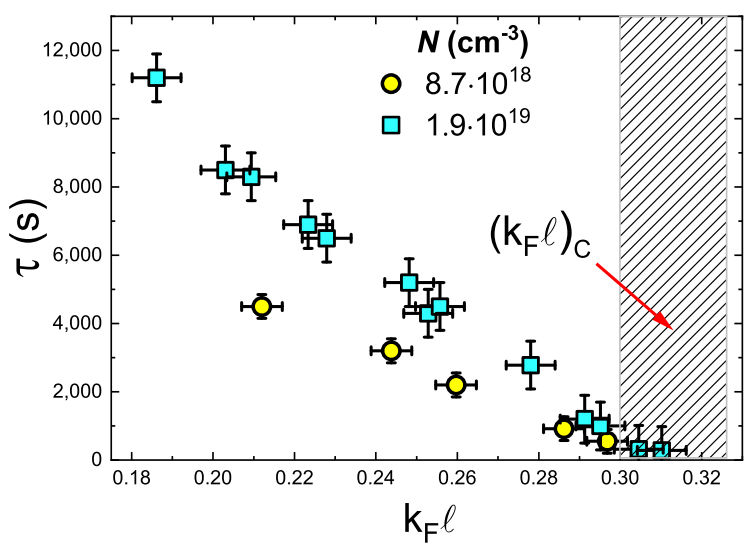

FIG. 10: The dependence of the relaxation times $\tau$ (as defined in Figs. 7 and 8 above) on the disorder parameter $\mathrm{k}_{\mathrm{F}} \ell$ near the critical-regime of the metal-to-insulator transition (marked by the hatched area).

\section{Disorder vs. Interaction}

To get a better picture of the dynamics we expanded a preliminary study of $\operatorname{In}_{\mathrm{x}} \mathrm{O}$ films with low carrierconcentration $N=8.7 \times 10^{19} \mathrm{~cm}^{-3}$ 25] by measuring 13 samples from the batch with $N=1.9 \times 10^{19} \mathrm{~cm}^{-3}$ with which most of the screen-reference samples studied here were made.

Figure 10 shows the relaxation time $\tau$ (defined by the exponential-relaxation regime of the gate-protocol) as function of the dimensionless parameter $\mathrm{k}_{\mathrm{F}} \ell$. As in other studies [26, 27], $\mathrm{k}_{\mathrm{F}} \ell=\left(3 \pi^{2}\right)^{2 / 3} \cdot \hbar \cdot \sigma_{\mathrm{RT}} \cdot e^{-2} \cdot N^{-1 / 3}$ was taken as the measure of the quenched disorder $\left(\sigma_{\mathrm{RT}}\right.$ is the sample conductivity at room-temperature).

There are two interesting features that emerge from the data; First, the relaxation-time decreases with $\mathrm{k}_{\mathrm{F}} \ell$ and tends to zero roughly at the disorder range where 
the system undergoes the metal to insulator transition. The critical value of disorder $\left(k_{\mathrm{F}} \ell\right)_{\mathrm{C}}$ for the metalinsulator transition was independently measured for two versions of the material with $N \approx 10^{21} \mathrm{~cm}^{-3}$ [26] and $N \approx 10^{19} \mathrm{~cm}^{-3}$ [27] yielding in both $\left(\mathrm{k}_{\mathrm{F}} \ell\right)_{\mathrm{C}}=0.31 \pm 0.03$.

That the glassy features end at the transition is an important finding; it supports the conjecture that the slow relaxation is an electronic effect rather than reflecting structural defects. Note that the reduction of $\tau$ with $\mathrm{k}_{\mathrm{F}} \ell$ is achieved in $\operatorname{In}_{\mathrm{x}} \mathrm{O}$ by thermal-annealing. Changes in the structural properties of the material during the annealing process were extensively studied in [17] by electrondiffraction, energy-dispersive spectroscopy, x-ray interferometry, and optical techniques. The study revealed that the change in the resistance from the as-deposited deeply-insulating state all the way to the metallic regime is mainly due to increase of the material density. In particular, the samples retained their amorphous structure and composition throughout the entire process. Moreover, the dynamics associated with structural changes monitored during annealing and recovery of the samples was qualitatively different than that of the electron-glass and did not change its character throughout the entire range of disorder. The diminishment of $\tau$ with $\mathrm{k}_{\mathrm{F}} \ell$ cannot then be identified with the elimination of some peculiar structural defects.

Secondly, the relaxation-time is not a function of just $\mathrm{k}_{\mathrm{F}} \ell$; it appears that it also depends on the carrierconcentration $N$. Indeed, the exponential relaxation regime (which allows an unambiguous definition of $\tau$ ) become quickly out of reach for samples when $N \geq 5 \times 10^{19} \mathrm{~cm}^{-3}$. The data in Fig.10 seem to suggest a scaling relation of the form:

$$
\tau=\tau(N) \cdot\left[\mathrm{k}_{\mathrm{F}} \ell-\left(\mathrm{k}_{\mathrm{F}} \ell\right)_{\mathrm{C}}\right] ; \text { for } \mathrm{k}_{\mathrm{F}} \ell \geq\left(\mathrm{k}_{\mathrm{F}} \ell\right)_{\mathrm{C}}
$$

where the prefactor $\tau(N)$ presumably increases with carrier-concentration. One may surmise that the dependence on $N$ may be the effect of interactions. The logic is based on the realization that, due to lack of electronic screening of the Anderson-insulator, higher density of carriers enhances the strength of interaction. While it is plausible that interactions in the localized system get stronger with $N$, it is not necessarily the main (or the only) reason for slower relaxation [17]. It is here that the issue of separating effects of Coulomb interaction from of the effect of disorder that presents a frustrating problem because interaction and disorder both increase with $N$. Actually, a viable cause for $\tau$ increasing with $N$ is the higher degree of disorder in samples that have higher carrier-concentrations. Note that a pre-condition for the electronic system to exhibit slow relaxation is Andersonlocalization [17]. This requires that the disorder energy $\mathcal{W}$ has to be larger than the Fermi energy $E_{\mathrm{F}}$ by a certain factor 28, 29]. All other things being equal, a system with larger carrier-concentration $N$ must be more disordered to be Anderson-localized and thus has larger $\mathcal{W}$.

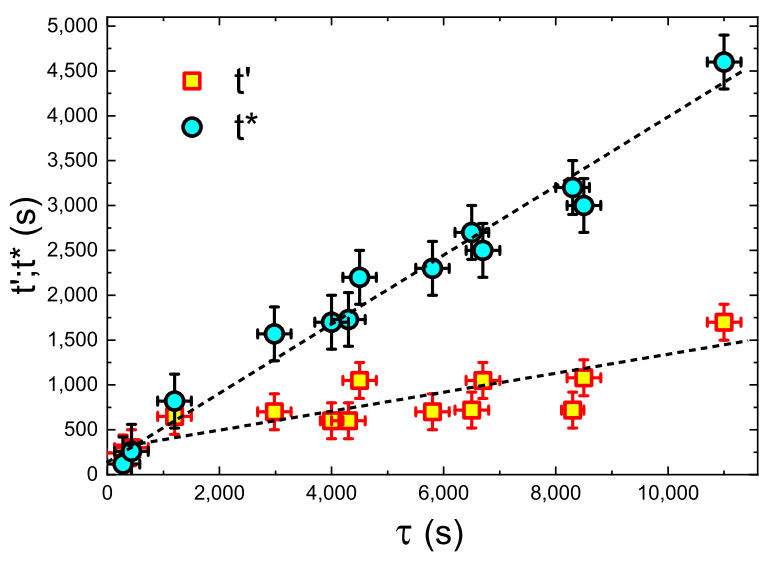

FIG. 11: The end-times for the logarithmic and simple exponential relaxation t' and $t^{*}$ respectively as a function of $\tau$ for the samples studied by the gate-protocol in Fig.10.

This, in turn, will exponentially slowdown the inter-site transitions, whether activated or through tunneling.

The way that Coulomb interactions affect thermalization dynamics is less clear. Interactions may modify transition rates through reduction of the density of states and many-particle transitions may be involved in the process but it is hard to find experimental evidence that may be uniquely related to these mechanisms. A large magnitude of memory-dip, suggestive of a more dominant role of interactions, is actually found in low- $N$ systems where dynamics is relatively fast as found in the present study. This however does not mean that interactions act to speed-up thermalization, rather it shows that the disorder effect (being weaker in low- $N$ ) is more important. A possible example for enhanced carrier-concentration without the accompanying increase of disorder was observed in GeSbTe samples in their persistent photoconductive state 30]. This caused an enhanced magnitude of the memory-dip, which was interpreted as an interaction effect 30]. It also slowed-down the dynamics but interaction is not the only possible mechanism for it; the slow decay of the photo-induced carriers may be the more mundane reason.

The transition to the exponential relaxation at long times, as observed for example in Fig.7 above, is expected. A $\log (\mathrm{t})$ relaxation is limited to intermediate times; it has to cross over to a different form for both short and long times [31]. The transition from a logarithmic to the exponential-relaxation regime is preceded by a more complicated time dependence which perhaps resembles the fast relaxation observed in phosphorousdoped silicon 32. This region extends over a time-period that grows monotonically with disorder [33] as is shown in Fig.11.

The dependence of the dynamics on $\mathrm{k}_{\mathrm{F}} \ell$ may be summarized as follows: As $\mathrm{k}_{\mathrm{F}} \ell$ increases and the system approaches the diffusive regime, the rate-distribution, 
that controls the relaxation from an excited state, gets narrower due to reduction of the lowest transition-rates (associated with the $\tau$ deduced from the exponentialrelaxation regime). Concomitantly, the range over which logarithmic relaxation is observed shrinks linearly with $\mathrm{k}_{\mathrm{F}} \ell$ (Fig.11).

It is intriguing that the time-period for the $\log (\mathrm{t})$ relaxation in electron-glass may extend over almost six decades 24] without a sign of a crossover. To account for such an extensive range one has to assume a fairly uniform distribution of transition-rates over a wide frequency range. It seems obvious that a main ingredient in the underlying mechanism is sufficiently strong disorder, but it probably also involves many-body effects [34]. The current study demonstrated that electronic relaxation extending over thousands of seconds is a viable possibility without the long-range part of the Coulomb interaction playing a significant part (and therefore the DOS at the Fermi-energy must be finite even at $\mathrm{T}=0$ ).

It is harder to assess the contribution of short and medium-range Coulomb interaction to the dynamics. One might argue that the faster dynamics observed as the system approaches the metallic regime may, at least in part, be due to the enhanced dielectric-constant that in turn weakens the interaction. The dielectric-constant in the localized state is expected to increase significantly near the transition [35]. However, the functional dependence of $\tau\left(\mathrm{k}_{\mathrm{F}} \ell\right)$ shown in Fig.10 does not exhibit a change from the linear dependence as the transition is approached. Therefore, this scenario is not supported by our experiments. Interactions are more likely to play a significant role in the ultra-slow processes that are necessary to reach the true ground-state of the system, a process that presumably hinges on many-particle transitions [36].

There are other mechanism that may contribute to stretch the transition-rates distribution and afford an extended $\log (\mathrm{t})$ dependence. Reduction of transitionrates relative to the "bare" rates controlled by disorder may occur for non-local interactions. These may bring into play additional constraints as well as effects related to coupling of the tunneling charge to other degrees of freedom (polaronic-effects, and the orthogonalitycatastrophe [37-39]). Resolution of these issues remain a challenge to theory.

Illuminating discussions with A. Vaknin and M. Schechter are gratefully acknowledged. This research has been supported by a grant No 1030/16 administered by the Israel Academy for Sciences and Humanities.

[1] P. W. Anderson, Phys. Rev. 109, 1492 (1958).

[2] L. Fleishman and P. W. Anderson, Phys. Rev. B 21, 2366 (1980).
[3] I. V. Gornyi, A. D. Mirlin, and D. G. Polyakov, Phys. Rev. Lett. 95, 206603 (2005).

[4] D. M. Basko, I. L. Aleiner, and B. L. Altshuler, Ann. Phys. (N.Y.) 321, 1126 (2006).

[5] V. Oganesyan and D. A. Huse, Phys. Rev. B 75, 155111 (2007); Rahul Nandkishore and David A. Huse, Annu. Rev. Condens. Matter Phys., 6, 15 (2015).

[6] M. Ben-Chorin, D. Kowal and Z. Ovadyahu, Phys. Rev. B 44, 3420 (1991); M. Ben Chorin, Z. Ovadyahu and M. Pollak, Phys. Rev. B48, 15025 (1993).

[7] A. Vaknin, Z. Ovadyahu, and M. Pollak, Phys. Rev. B 65, 134208 (2002).

[8] J. H. Davies, P. A. Lee, and T. M. Rice, Phys. Rev. Lett, 49, 758 (1982); M. Grünewald, B. Pohlman, L. Schweitzer, and D. Würtz, J. Phys. C, 15, L1153 (1982); J. H. Davies, P. A. Lee, and T. M. Rice, Phys. Rev. B 29, 4260 (1984); C. C. Yu, Phys. Rev. Lett., 82, 4074 (1999).

[9] M. Müller and L. B. Ioffe, Phys. Rev. Lett. 93, 256403 (2004)

[10] Vikas Malik and Deepak Kumar, Phys. Rev. B 69, 153103 (2004).

[11] R. Grempel, Europhys. Lett., 66, 854 (2004); A. B. Kolton, D. R. Grempel, and D. Dominguez, Phys. Rev. B 71, 024206 (2005).

[12] Eran Lebanon, and Markus Müller, Phys. Rev. B 72, 174202 (2005); M. Müller and E. Lebanon, J. Phys. IV France, 131, 167 (2005).

[13] Ariel Amir, Yuval Oreg, and Yoseph Imry, Phys. Rev. B 77, 165207 (2008); Ariel Amir, Yuval Oreg, and Yoseph Imry, Annu. Rev. Condens. Matter Phys. 2, 235 (2011).

[14] Y. Meroz, Y. Oreg and Y. Imry, EPL, 105, 37010 (2014).

[15] M. Pollak, M. Ortuño and A. Frydman, "The Electron Glass", Cambridge University Press, England (2013).

[16] M. Pollak, Discuss. Faraday Soc. 50, 13 (1970); A. L. Efros and B. I. Shklovskii, J. Phys. C: Solid State Phys., 8, L49 (1975).

[17] Z. Ovadyahu, Phys. Rev. B. 95, 134203 (2017).

[18] B. Hadley, M. Green, M. Pollak, R. Chicon, and M. Ortuño, J.l of Non-Crys. Solids 97\&98, 233 (1987).

[19] Z. Ovadyahu, Phys. Rev. B 95, 214207 (2017).

[20] Y. Imry and Z. Ovadyahu, Phys. Rev. Lett.. 49, 841 (1982).

[21] B. L. Altshuler, A. G. Aronov, and A. Yu. Zyuzin, Sov. Phys. JETP 59, 415 (1984); Zh. Eksp. Teor. Fiz. 86, 709 (1984).

[22] A. I. Larkin and D. E. Khmel'nitskii, Sov. Phys. JETP 56, 647 (1982); Zh. Eksp. Teor. Fiz. 83, 1140 (1982).

[23] A. Frydman and Z. Ovadyahu, Phys. Rev. B 55, 9047 (1997).

[24] Z. Ovadyahu, and M. Pollak, Phys. Rev. B 68, 184204 (2003).

[25] Z. Ovadyahu, Phys. Rev. B 97, 214201 (2018).

[26] D. Shahar and Z. Ovadyahu, Phys. Rev. B 46, 10917 (1992).

[27] U. Givan and Z. Ovadyahu, Phys. Rev. B 86, 165101 (2012).

[28] B. R. Bulka, B. Kramer, and A. MacKinnon, Z. Phys. B - Condensed Matter 60, 13 (1985).

[29] B. Bulka, M. Schreiber, and B. Kramer, Z. Phys. B Condensed Matter 66, 21 (1987).

[30] Z. Ovadyahu, Phys. Rev. Lett., 115, 046601 (2015); Z. Ovadyahu, Phys. Rev. B 97, 054202 (2018).

[31] G. Mihàly and L. Mihàly, Phys. Rev. Lett., 52, 149 
(1984).

[32] V. K. Thorsmølle, and N.P. Armitage, Phys. Rev. Lett. 105, 086601 (2010).

[33] This part of the relaxation may be fitted to a stretchedexponential function, $\Delta \mathrm{G}(\mathrm{t})=\mathrm{A} \cdot \exp \left[-\left(\mathrm{t} / \tau^{\prime}\right)^{\beta}\right]$ by adjusting the parameters $\tau^{\prime}$ and $\beta$. For example, the data for the intermediate range of $15 \mathrm{~s} \leq \mathrm{t} \leq 5 \times 10^{3} \mathrm{~s}$ in Fig. $7 \mathrm{c}$ may be fitted with the three parameters $\mathrm{A}=3.3 \times 10^{-8} \Omega^{-1}, \tau^{\prime}=185 \mathrm{~s}$, and $\beta=0.33$.

[34] M. Pollak and M. Ortuño, Sol. Energy Mater., 8, 81 (1982); M. Pollak, Phil. Mag. B 50, 265 (1984).

[35] T. G. Castner, N. K. Lee, G. S. Cieloszyk, and G. L. Salinger, Phys. Rev. Lett. 34, 1627 (1975); David Stroud and David J. Bergman, Phys. Rev. B 25, 2061(R) (1982); H. S. Choi, J. S. Ahn, J. H. Jung, T. W. Noh, and D. H.
Kim, Phys. Rev. B 54, 4621 (1996).

[36] S. D. Baranovskii, B. I. Shklovskii, A. L. Efros, Sov. Phys. JETP 51, 199 (1980); A. Möbius, M. Richter, and B. Drittler, Phys. Rev. B 45, 11568 (1992); A. L. Efros, B. Skinner, and B. I. Shklovskii, Phys. Rev. B 84, 064204 (2011).

[37] P. W. Anderson, Phys. Rev. Lett. 18, 1049 (1967); A. J. Leggett et al., Rev. Mod. Phys. 59, 1 (1987); Z. Ovadyahu, Phys. Rev. Lett., 99, 226603 (2007).

[38] Vedika Khemani, Rahul Nandkishore, and S. L. Sondhi, Nature Physics, 11, 560 (2015).

[39] D. L. Deng, J. H. Pixley, X Li, S. D. Sarma, Phys. Rev. B. 92, 220201(R) (2015). 